\title{
A 3-year prospective study of the effects of adjuvant treatments on cognition in women with early stage breast cancer
}

\author{
V Jenkins*, , V Shilling', G Deutsch ${ }^{2}$, D Bloomfield ${ }^{2}$, R Morris', S Allan ${ }^{3}$, H Bishop ${ }^{4}$, N Hodson ${ }^{2}$, S Mitra $^{2}$, \\ G Sadler ${ }^{5}$, E Shah ${ }^{5}$, R Stein ${ }^{6}$, S Whitehead ${ }^{5}$ and J Winstanley ${ }^{4}$ \\ 'Cancer Research UK Psychosocial Oncology Group, Brighton and Sussex Medical School, University of Sussex, East Sussex BNI 9QG, UK; ${ }^{2}$ Sussex \\ Cancer Centre, Royal Sussex County Hospital, Eastern Road, Brighton, East Sussex BN2 5BE, UK; ${ }^{3}$ Eastbourne District Hospital, Kings Drive, Eastbourne, \\ East Sussex BN2I 2UD, UK; ${ }^{4}$ Royal Bolton Hospital, Minerva Road, Farnworth, Bolton BL4 OJR, UK; ${ }^{5}$ Conquest Hospital, The Ridge, St Leonards-on-Sea, \\ East Sussex TN37 7RD, UK; ${ }^{6}$ Ludwig Institute for Cancer Research, Courtauld Building, 91 Riding House Street, London WIW 7BS, UK
}

The neuropsychological performance of 85 women with early stage breast cancer scheduled for chemotherapy, 43 women scheduled for endocrine therapy and/or radiotherapy and 49 healthy control subjects was assessed at baseline (TI), postchemotherapy (or 6 months) (T2) and at 18 months (T3). Repeated measures analysis found no significant interactions or main effect of group after controlling for age and intelligence. Using a calculation to examine performance at an individual level, reliable decline on multiple tasks was seen in $20 \%$ of chemotherapy patients, $26 \%$ of nonchemotherapy patients and I $8 \%$ of controls at T2 (I8\%, 14 and II\%, respectively, at T3). Patients who had experienced a treatment-induced menopause were more likely to show reliable decline on multiple measures at $\mathrm{T} 2(\mathrm{OR}=2.6,95 \%$ confidence interval $(\mathrm{Cl}) 0.823-8.266 \mathrm{P}=0.086)$. Psychological distress, quality of life measures and self-reported cognitive failures did not impact on objective tests of cognitive function, but were significantly associated with each other. The results show that a few women experienced objective measurable change in their concentration and memory following standard adjuvant therapy, but the majority were either unaffected or even improve over time. British Journal of Cancer (2006) 94, 828-834. doi: I 0.1038/sj.bjc.6603029 www.bjcancer.com

Published online 7 March 2006

(c) 2006 Cancer Research UK

Keywords: breast cancer; chemotherapy; endocrine treatment; cognitive dysfunction

There are various chemotherapy regimens in use to treat early stage breast cancer, some more toxic than others, yet two women receiving the same combination of drugs may feel completely different during the course of their treatment. Whereas a number of patients may experience few side effects and are able to continue with their usual activities, others' lives are significantly disrupted. One of the growing concerns among patients, clinicians, neuropsychologists and other health professionals is that adjuvant treatments for breast cancer may affect cognition.

It is important to recognise that the 'cognitive dysfunction' associated with chemotherapy is not as severe as that found with acute amnesia or presenile dementia. Rather it is more usual for women to complain of feelings of 'fuzzy headedness' or 'mental slowness', sometimes described as 'chemo-fog' (Schagen et al, 2002). Evidence from the existing literature suggests that around $16-75 \%$ of breast cancer patients receiving high and standard dose chemotherapy experience some degree of cognitive dysfunction (Wieneke and Dienst, 1995; Tchen et al, 2003). Hypotheses to explain these changes include, chemotherapy having a direct toxic effect on the brain, changes in circulating hormones particularly in those women who experience a treatment-induced menopause

*Correspondence: Dr V Jenkins; E-mail: val@sussex.ac.uk Received 23 November 2005; revised I February 2006; accepted 3 February 2006; published online 7 March 2006 following chemotherapy, plus fatigue, anxiety and depression (McAllister et al, 2004).

Objective cognitive impairment is not apparent in all women, yet subjectively many women report changes in their memory and attention (Shilling et al, 2005a). Much of the earlier published objective data were derived from cross-sectional studies, with only two containing pretreatment (baseline) assessments (Wefel et al, 2004; Bender et al, 2005). In the former, a third of patients were reported to have cognitive impairment at baseline in one or more domains and this figure increased to $61 \%$ postchemotherapy, with $50 \%$ improving by the 12 -month assessment. In the latter study, women who received chemotherapy showed impairment on a verbal memory measure, whereas those who had received chemotherapy plus tamoxifen declined on visual, verbal and working memory. Most of these differences were detected by the 18 -month assessment. Both studies are limited, however, by their small sample size ( $n=18$ and $n=22$ at completion of study, respectively), making meaningful conclusions difficult. A more recent preliminary analysis of 50 chemotherapy patients with 43 healthy controls showed that at the postchemotherapy assessment, $34 \%$ of patients compared with $19 \%$ of healthy control subjects experienced reliable cognitive decline relative to baseline performance on multiple measures (Shilling et al, 2005a). Of interest was that eight out of $17(47 \%)$ of these patients experienced a treatment-induced menopause following chemotherapy.

The potential effect of endocrine therapy on cognitive function is more difficult to assess because of confounding factors. Women 
with breast cancer who receive only endocrine treatment tend to be postmenopausal and therefore older than those who receive chemotherapy. One cross-sectional study of cognitive function in women who participated in the Arimidex and Tamoxifen, Alone or in Combination (ATAC) trial noted that verbal memory and processing speed were impaired compared to healthy postmenopausal women (Jenkins et al, 2004). Another study reported that tamoxifen users consulted their physician about memory problems more often than nonusers (Paganini-Hill and Clark, 2000), but as yet no study has reported longitudinal data on the effects of either tamoxifen or aromatase inhibitors on memory and attention.

Establishing the prevalence, clinical significance and impact on quality of life of cognitive impairment following breast cancer treatments is important. At the moment, the literature is too confusing to permit clear discussion. There is also some concern that the problems have been overestimated, particularly in the light of a recent meta-analysis (Falleti et al, 2005), which suggests that although statistically significant, the magnitude of effect sizes previously reported is small to moderate ranging from -0.03 to -0.51 of a standard deviation below controls. This report presents results from a prospective longitudinal study of the impact of breast cancer treatments on the cognitive functioning of 128 women at three time points.

\section{MATERIALS AND METHODS}

\section{Participants}

Women with early breast cancer from hospitals across the UK were invited to join the study following surgery but before the start of adjuvant therapy. Exclusion criteria included advanced disease, previous treatment for any cancer, receiving neo-adjuvant chemotherapy, those with a previous history of stroke dementia, degenerative disease and alcohol or drug abuse. Of 224, $153(68 \%)$ patients were recruited to the study; of the 69 who did not, 39 were not interested, 11 were not eligible owing to age ( $>75$ years) or previous treatment history and 19 did not complete the baseline assessment before the start of treatment. The control group was a sample of convenience made up of friends and family of the patients and experimenters and from a local women's group.

Of 153,100 women were scheduled to receive adjuvant chemotherapy and 53 to receive radiotherapy and/or endocrine therapy or no further treatment. Data are presented on 128 breast cancer patients (85 received chemotherapy, $43 \mathrm{did}$ not) and 49 healthy controls. Of the 15 chemotherapy patients (five no longer wanted to take part, four could not be contacted, one owing to ill-health (unrelated), one owing to disease progression and four had died of their disease); 10 nonchemotherapy patients (four no longer wanted to take part, one was not contactable, two withdrew for unrelated health reasons, two because of disease progression and one had died of causes unknown to the authors) and three healthy controls (one for health reasons, one for family reasons and one emigrated) did not complete all assessments. A further six healthy controls were excluded prior to analyses in order to bring the groups into closer alignment for age and full-scale intelligence. The study had local ethics committee approvals and all participants gave full written consent.

Table 1 shows the characteristics for the three participant groups. The nonchemotherapy group differed significantly from the healthy controls and chemotherapy group by age $(F=22.74$, $P<0.0001 ; F=20.09, P<0.0001$, respectively) and from the healthy control group by years spent in full time education $(F=5.58$, $P=0.02)$. These differences are accounted for in the analyses. The chemotherapy group were less likely than the other groups to be peri- or postmenopausal at baseline $\left(\chi^{2}=4.90, P=0.027\right.$; $\chi^{2}=10.87, P=0.001$ for healthy controls and nonchemotherapy, respectively) and the nonchemotherapy and control groups were not more likely to have used HRT.

Table 2 details the tumour grade and type of surgery for both patient groups and chemotherapy regimen. In total, $59 \%$ of the chemotherapy and $14 \%$ of the nonchemotherapy patients were lymph node positive. Time between surgery and baseline assessment ranged from 21 to 83 days (mean 41.29 s.d. 13.30) in the chemotherapy group (date of surgery missing for six patients) and 22 to 92 days in the nonchemotherapy group (mean 53.21s.d. 16.03 ). Eighty eight percent (75 out of 85 ) of the chemotherapy and $74 \%$ (32 out of 43 ) of the nonchemotherapy patients were seen within 2 weeks of their second assessment and at T3, the proportions were $91 \%$ (77 out of 85 ) and $86 \%$ (37 out of 43 ), respectively.

At T1, 40 out of 43 (93\%) nonchemotherapy patients had started endocrine therapy; 36 received tamoxifen and four anastrozole (one went on to have goserelin injections, six switched endocrine treatment during the study and one ceased endocrine treatment) and between T1 and T2 assessments, 36 out of $43(84 \%)$ had completed a course of radiotherapy. By T2, 20 out of $85(23 \%)$ chemotherapy patients had started endocrine therapy; 17 received tamoxifen, three letrozole and by T3, this had risen to 60 (71\%); 46 tamoxifen, nine letrozole and five anastrozole (two patients switched from tamoxifen to anastrozole between assessments T2

Table I Age (at baseline), IQ, years in education and psychological distress

\begin{tabular}{|c|c|c|c|}
\hline & Chemotherapy $(N=85)$ & Nonchemotherapy $(N=43)$ & Control $(N=49)$ \\
\hline Age (years) & $51.49(9.57)$ & $58.93(7.27)$ & $51.90(6.87)$ \\
\hline $\mathrm{IQ}$ & $109.89(12.34)$ & $111.05(11.24)$ & $112.04(9.14)$ \\
\hline \multicolumn{4}{|l|}{ Menopausal status } \\
\hline Premenopausal & 39 (46\%) & $7(16 \%)$ & $13(26 \%)$ \\
\hline Peri/Postmenopausal & $46(54 \%)$ & $36(84 \%)$ & $36(73 \%)$ \\
\hline \multicolumn{4}{|l|}{ HRT use } \\
\hline Never & 18 (39\%) & $13(36 \%)$ & 14 (39\%) \\
\hline Current & & & $15(42 \%)$ \\
\hline Past & $28(61 \%)$ & $23(64 \%)$ & $7(19 \%)$ \\
\hline \multicolumn{4}{|c|}{ Above threshold $G H Q_{12}$ scores $^{\mathrm{a}, \mathrm{b}}$} \\
\hline TI & $46(55 \%)$ & $26(62 \%)$ & $8(16 \%)$ \\
\hline $\mathrm{T} 2$ & $43(51 \%)$ & $10(24 \%)$ & $9(18 \%)$ \\
\hline
\end{tabular}

${ }^{a}$ Data missing from I chemotherapy patient at $\mathrm{TI}$ and $\mathrm{T} 2$ and from 7 at T3. ${ }^{\mathrm{b}}$ Data missing from one nonchemotherapy patient at each time point. 
Table 2 Treatment details for both patient groups

\begin{tabular}{|c|c|c|}
\hline & $\begin{array}{l}\text { Chemotherapy } \\
\text { group }(n=85)\end{array}$ & $\begin{array}{c}\text { Nonchemotherapy } \\
\text { group }(n=43)\end{array}$ \\
\hline \multicolumn{3}{|l|}{ Tumour grade } \\
\hline Grade I & 4 & 16 \\
\hline Grade 3 & 31 & 25 \\
\hline Grade 3 & 50 & 2 \\
\hline \multicolumn{3}{|l|}{ Type of surgery } \\
\hline WLE & 47 & 36 \\
\hline Mastectomy & 26 & 7 \\
\hline $\begin{array}{l}\text { Mastectomy and } \\
\text { reconstruction }\end{array}$ & 7 & i \\
\hline Bilateral mastectomy & 4 & \\
\hline Missing & I & \\
\hline \multicolumn{3}{|l|}{ Type of chemotherapy } \\
\hline $\mathrm{FEC}^{\mathrm{a}} \times 6$ cycles & 51 & \\
\hline FEC $^{\mathrm{b}} \times 8$ cycles & 8 & \\
\hline CMF $\times 6$ cycles & 2 & \\
\hline 4FEC 4 docetaxel $\times 8$ cycles & 3 & \\
\hline AC $\times 4$ cycles & 4 & \\
\hline EC $\times 6$ cycles & 9 & \\
\hline 4EC 4 paclitaxel $\times 8$ cycles & 5 & \\
\hline $4 \mathrm{E} 4 \mathrm{CMF} \times 8$ cycles & I & \\
\hline 4E 4FEC $\times 8$ cycles & I & \\
\hline
\end{tabular}

${ }^{\mathrm{a}}$ Data missing from one patient. ${ }^{\mathrm{b}}$ Data missing from one patient.

and T3, and two received trastuzumab). Of the patients, $16(19 \%)$ had started a course of radiotherapy by T2 and by T3, $71(83.5 \%)$ had completed radiotherapy treatment. Out of 39, 32 premenopausal chemotherapy patients experienced a treatment-induced menopause.

\section{Assessments}

Cognitive assessments were made at baseline (T1), 4 weeks after the final chemotherapy session (six months in the other groups) (T2), and 12 months after the final chemotherapy session (18 months in the other groups) (T3).

The cognitive test battery assesses several broad areas of cognitive function as outlined in Figure 1. The tasks used are sensitive and have shown changes in a number of patient groups, including those suffering with AIDS, Parkinson's disease, head injury, and following cardiac surgery. They can provide information on a variety of cognitive processes including attention, learning, memory, planning and organisational strategies.

The tests were administered in the same order following the requirements of the Wechsler Memory Scale-III (Weschler, 1998). All participants were screened for dementia, using the information and orientation subtest of the WMS III. The battery of standardised neuropsychological tests is briefly described below.

\section{Intelligence}

Intelligence was assessed using the National Adult Reading Test (Nelson, 1991). Wechsler Adult Intelligence Scale FSIQ was predicted from this score.

\section{Verbal memory}

WMS III logical memory part 1 and 2 indicate: immediate and delayed recall of a short paragraph, respectively. Rey Auditoryverbal learning test (Rey, 1964) is a word list-learning task consisting of five verbal presentations with recall of a 15-word list.

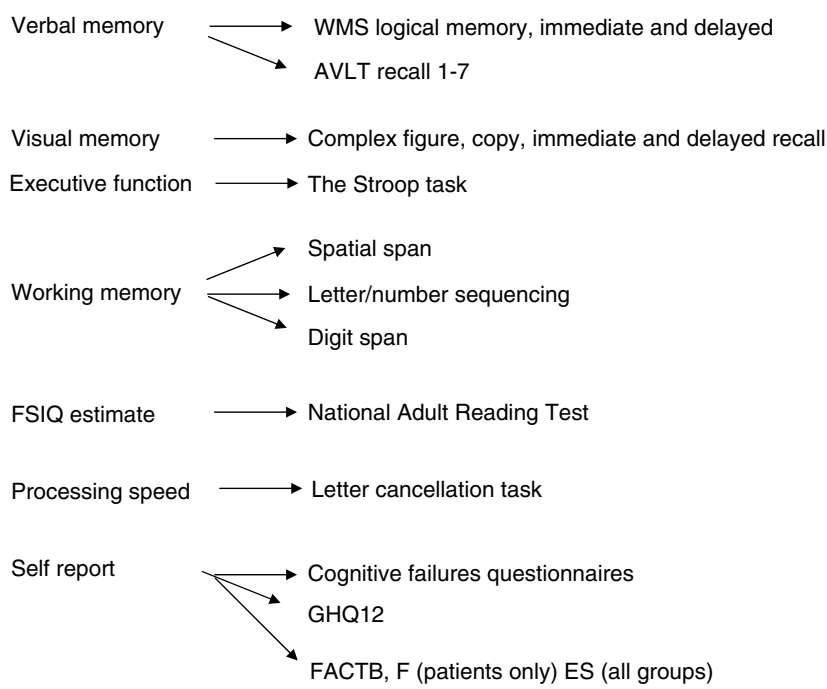

Figure I Cognitive test battery.

Three scores are reported from this test: supraspan score (number of words recalled from the first presentation of the list), total recall score (total words recalled from the first five presentations) and delayed recall score (total words recalled after half an hour delay).

\section{Visual memory}

Complex figure task with two alternate forms (Rey, 1941) (Taylor, 1979): copy, immediate and delayed recall of a complex geometric figure.

\section{Working memory}

WMS III letter-number sequencing: sequences of letters and numbers must be reordered giving numbers first in ascending order and then letters in alphabetical order. WMS III digit span: strings of digits must be repeated in the same and then in the reverse order to presentation. WMS III spatial span: spatial patterns must be reproduced first in the same and then in the reverse order to presentation.

\section{Executive function}

The Stroop task (Golden, 1978) has three conditions, colour word reading, colour patch naming and the interference condition in which colour words are printed in incompatible ink colour. The participant names the colour of the ink, requiring the inhibition of the more salient word reading.

\section{Processing speed and vigilance}

Processing speed and vigilance are assessed using a letter cancellation task. A composite score is calculated based on both speed and accuracy.

\section{Self-report measures}

All participants completed the General Health Questionnaire 12 $\left(\mathrm{GHQ}_{12}\right)$ and the Broadbent cognitive failures questionnaire (Broadbent et al, 1982). The $\mathrm{GHQ}_{12}$ is a 12 -item general health measure designed to screen for probable, nonpsychotic psychiatric disorder in community and medical settings (Goldberg and 
Williams, 1988). The cognitive failures questionnaire comprises a series of 25 questions relating to lapses in attention in everyday life, such as forgetting what the person went into a room to do. Questions are rated on a five-point scale ranging from 0-'never' to 5-'very often'. As part of a structured interview (data to be presented elsewhere), patients were also asked at T2 and T3 whether they had noticed any changes in their memory and attention. Patients completed the Functional Assessment of Cancer Therapy questionnaire (Breast) (FACT B) (Brady et al, 1997) and the fatigue (F) subscale (Yellen et al, 1997). In addition, all participants completed a quality of life measure of endocrine symptoms (ES) (Fallowfield et al, 1999).

\section{Statistical methods}

The Statistical Package for the Social Sciences (SPSS) version 11.5 was used for all statistical analyses. Baseline cognitive performance on each measure was analysed using stepwise multiple regression with the predictor variables of treatment group, age, FSIQ, education, psychological distress and menopausal status. Group comparisons on cognitive performance on each measure and on self-reported cognitive failures were made at the three time points, using repeated measures ANOVA with group as the betweensubjects factor and time point as the within-subject factor. Where significant main effects of group were found, any variable that had significantly predicted baseline performance on that task was covaried.

Such group comparisons do not identify impairment in subgroups of the population or account for practice effects. To examine performance at an individual level, we used the reliable change index (RCI) with a correction for observed practice effects on each measure (Sawrie et al, 1996). By using the method put forward by Jacobson and Truax (1991), an RCI was calculated for each cognitive measure using the baseline and $\mathrm{T} 2$ and baseline and T3 data of the control subjects (see Appendix 1).

\section{RESULTS}

The result section is divided into changes in performance within and between the groups over time, followed by the proportion of individuals whose performance either improved or declined over time for each task, compared with their baseline performance. The results from these objective cognitive tasks are then compared with the patients' subjective measures of changes in psychological distress, memory and concentration and quality of life.

\section{Group comparisons}

Baseline (T1) Combinations of education, age and FSIQ consistently predicted cognitive performance (see Table 3 ). Treatment group failed to significantly predict cognitive performance on any of the 14 measures independently of factors such as age, education and FSIQ, as did menopausal status and $\mathrm{GHQ}_{12}$ scores. In all cases, the regression was a poor fit describing between 8 and $22 \%$ of the variance ( $R^{2}$ adj between 8 and $\left.20 \%\right)$, but the overall relationship was significant $(P<0.0001)$ in all cases.

Repeated measures ANOVA Repeated measures ANOVAs with group (chemo vs nonchemo vs control) as the between-subject factor and time point (T1 vs T2 vs T3) as the within-subject factor were conducted for each of the 14 cognitive measures. In a number of cases, the assumption of sphericity was violated. In all cases $\epsilon$ was greater than 0.75 , so the Huynh and Feldt correction was used. Bonferroni was used for post hoc comparisons where sphericity was violated; Games-Howell was used where sphericity was not violated.

Table 3 Results of regression analyses on baseline scores shows that combinations of education, age and Full Scale Intelligence Quotient (FSIQ) consistently predicted performance on the cognitive tasks

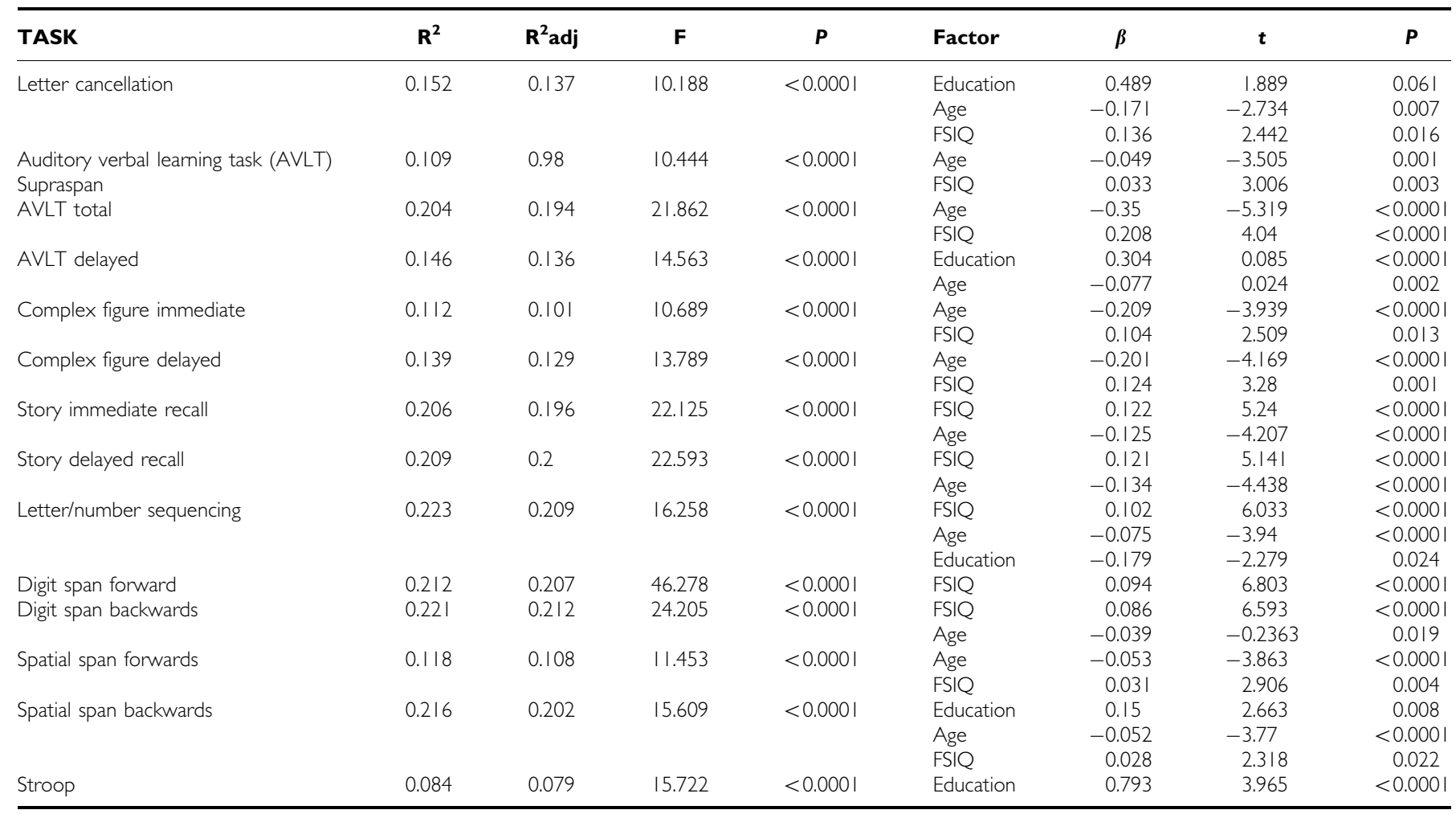


None of the measures showed a significant group $\times$ time point interaction. Six measures showed a significant main effect of time, with performance increasing linearly (letter cancellation $F=15.57 P<0.0001$; AVLT supraspan $F=4.083, P=0.018$; AVLT total $F=3.175, \quad P=0.043$; complex figure immediate recall $F=6.99=0.002 ; \quad$ complex figure delayed recall $F=10.69$, $P<0.0001$; digit span backwards $F=3.53, P=0.031$ and Stroop $F=4.29, P=0.014$ ).

Five measures showed a significant main effect of group: AVLT supraspan $(F=5.60, P=0.004)$; complex figure immediate recall $(F=3.79 P=0.024)$; complex figure delayed recall $(F=3.55$, $P=0.031)$; spatial span forwards $(F=3.72, P=0.026)$ and spatial span backwards $(F=3.71, P=0.026)$. Post hoc comparisons revealed significant differences were between the nonchemotherapy patient group and the healthy control group $(P=0.004 ; 0.021$; $0.026 ; 0.039 ; 0.036$ respectively). These five repeated measures ANOVAs were recalculated covarying factors that were significant predictors of performance at baseline (age, FSIQ and in the case of spatial span backwards this also included years in education). Group ceased to be a significant main effect after covarying these baseline predictor variables, although a trend towards an effect of group remained for AVLT supraspan $(F=2.68, P=0.071)$.

Reliable change analyses The proportion of each group showing reliable decline or reliable improvement for each task is shown for both time points in Table 4. Group did not significantly predict reliable decline on any individual measure at either time with the exception of spatial span forward at T2, which was more common in the healthy control group than in either patient group (overall $\left.\chi^{2}=13.44, P=0.001\right)$. Those who showed reliable decline were not significantly older, or less intelligent; nor were they more likely to have above threshold $\mathrm{GHQ}_{12}$ scores. Reliable improvement on two or more measures was not more common in any one group at T2, but at T3 multiple improvement was more likely in the patient groups, OR $2.78\left(\chi^{2}=4.925, P=0.02\right)$ and $2.54\left(\chi^{2}\right.$, n.s. $)$ for chemotherapy and nonchemotherapy, respectively Table 5.

The effect of endocrine therapy It was not possible to compare the effects of endocrine therapy on cognitive performance within the nonchemotherapy group as $91 \%$ were receiving endocrine therapy. Within the chemotherapy group, performance did not differ significantly on any measure between those who did or did not receive endocrine therapy at $\mathrm{T} 3$ ( $N=60$ and 25 , respectively).

Psychological distress $\left(G H Q_{12}\right)$ Table 1 shows the proportion of each group with above threshold $\mathrm{GHQ}_{12}$ scores, suggesting probable psychiatric morbidity at each time point. In both patient groups, rates of psychological distress were above $50 \%$ at baseline. In the nonchemotherapy group, this dropped significantly by $\mathrm{T} 2$ to levels approximately the same as the control group, where it remained at T3. In the chemotherapy group psychological distress remained high at T2 and did not drop to control levels until T3.

Self-reported cognitive failures Patients and controls reported similar numbers of cognitive failures in everyday life on the Broadbent Cognitive Failures questionnaire. Repeated measures ANOVA with time point as the within-subject factor and group as the between-subject factor found no significant main effect of group but a significant main effect of time point $(F=16.02$, $P<0.0001)$ and a significant interaction $(F=4.26, P=0.016)$. The scores show a different pattern for the three groups. In the healthy control group, scores were stable across the three time points (mean score 40.14, 40.41 and 40.08). In the chemotherapy group mean, scores were initially lower than in the healthy control group (37.95), rising significantly at T2 to $43.13(t=-4.24, P<0.0001)$ before dropping to 41.56 . Finally, the nonchemotherapy group also had mean scores lower than the healthy control group at baseline (37.64) but showed a steady increase over time with an overall

Table 4 Percentage of each group showing reliable decline, reliable improvement or no change for each task at T2 (T3)

\begin{tabular}{|c|c|c|c|c|c|c|c|c|c|}
\hline & \multicolumn{3}{|c|}{ Chemotherapy group $(n=85)$} & \multicolumn{3}{|c|}{ Nonchemotherapy group $(n=43)$} & \multicolumn{3}{|c|}{ Healthy control group $(n=49)$} \\
\hline & Decline & Improve & Stable & Decline & Improve & Stable & Decline & Improve & Stable \\
\hline Letter $^{\mathrm{a}}$ cancellation & $4(6)$ & $2(2)$ & $94(92)$ & $0(9)$ & $5(5)$ & $95(86)$ & $4(2)$ & $4(4)$ & $92(94)$ \\
\hline AVLT supraspan & $15(14)$ & $10(9)$ & 75 (77) & $9(5)$ & $9(5)$ & $82(90)$ & $8(6)$ & $16(6)$ & $76(88)$ \\
\hline AVLT total & $12(12)$ & $4(6)$ & $84(82)$ & $9(16)$ & $5(12)$ & $86(72)$ & $6(8)$ & $4(6)$ & $90(86)$ \\
\hline AVLT delayed & $5(5)$ & $2(5)$ & $93(90)$ & $2(0)$ & $12(12)$ & $86(88)$ & $6(0)$ & $4(6)$ & $90(94)$ \\
\hline Complex figure imm. & $5(5)$ & $8(12)$ & $87(83)$ & $12(2)$ & $5(13)$ & $84(85)$ & $4(4)$ & $2(4)$ & $94(92)$ \\
\hline Story del. & $10(1)$ & $15(8)$ & $75(91)$ & $9(5)$ & $9(5)$ & $82(90)$ & $2(2)$ & $4(4)$ & $94(94)$ \\
\hline Letter/number sequencing & $5(10)$ & $3(5)$ & $92(85)$ & $14(9)$ & $0(2)$ & $86(89)$ & $6(8)$ & $8(4)$ & $86(88)$ \\
\hline Digit span forward & $6(4)$ & $3(2)$ & $91(94)$ & $7(2)$ & $0(5)$ & $93(93)$ & $10(4)$ & $2(4)$ & $88(92)$ \\
\hline Digit span backwards & $2(5)$ & $0(3)$ & $98(92)$ & $7(5)$ & $0(9)$ & $93(86)$ & $8(2)$ & $4(2)$ & $88(96)$ \\
\hline Spatial span forwards & $0(2)$ & $3(18)$ & $97(80)$ & $0(7)$ & $5(9)$ & $95(84)$ & $10(6)$ & $4(0)$ & $86(94)$ \\
\hline Spatial span backwards & $5(4)$ & $3(1)$ & $92(95)$ & $5(0)$ & 7 (2) & $88(98)$ & $2(6)$ & $8(2)$ & $90(92)$ \\
\hline Stroop e,f & $11(4)$ & $5(8)$ & $84(88)$ & $9(7)$ & $2(9)$ & $88(84)$ & $6(4)$ & $6(4)$ & $88(92)$ \\
\hline
\end{tabular}

Missing data. ${ }^{a}$ Time 3; data missing from one chemotherapy patient and one nonchemotherapy patient. ${ }^{\mathrm{b}}$ Time 2 ; data missing from one healthy control. ${ }^{\mathrm{c}}$ Time 3 ; data missing from one chemotherapy patient and one healthy control. ${ }^{\mathrm{d}}$ Time 3; data missing from one chemotherapy patient. ${ }^{\mathrm{e}}$ Time 2 ; data missing from one chemotherapy patient and one healthy control. ${ }^{\mathrm{f}}$ Time 3; data missing from two chemotherapy patients and one healthy control.

Table 5 Extent of reliable change at T2 and T3

T2

\begin{tabular}{|c|c|c|c|c|c|}
\hline Chemo & Nonchemo & Control & Chemo & Nonchemo & control \\
\hline $1720 \%$ & I $25.6 \%$ & $918.4 \%$ & $1518.1 \%$ & $6 \mid 4.3 \%$ & $510.6 \%$ \\
\hline $1922.4 \%$ & $716.3 \%$ & $816.3 \%$ & $2732.1 \%$ & $1330.2 \%$ & $714.6 \%$ \\
\hline
\end{tabular}


significant increase $(F=13.34, \quad P=0.001)$ but no significant increase between consecutive time points.

At each time point, participants with above threshold $\mathrm{GHQ}_{12}$ scores reported significantly more cognitive failures than those with below threshold scores $(t=3.397, P=0.001 ; t=3.81$, $P<0.0001 ; t=3.22, P=0.002$ ), but self-reported cognitive failures did not significantly correlate with objective cognitive test scores. At interview, the majority of the chemotherapy group reported that they had noticed changes in their memory $(83 \%)$ and concentration $(80 \%)$ at $\mathrm{T} 2$. This fell to 60 and $45 \%$, respectively, by T3. The incidence was lower in the nonchemotherapy group with 45 and $38 \%$, respectively, noticing changes in memory and concentration at T2. However, at $\mathrm{T} 3$ the proportion reporting memory problems had risen to $59 \%$, although concentration problems remained lower at $37 \%$. Reporting of problems was significantly associated with $\mathrm{GHQ}_{12}$ threshold scores but not with objective measures of cognition.

Quality of life and cognitive function There was no significant main effect between the two patient groups on the fatigue subscale; however, there was a significant interaction $(F=3.91, P=0.021)$ and a significant main effect of time $(F=5.99, P=0.03)$. Although fatigue levels in the nonchemotherapy group were stable at all three times, the chemotherapy group showed a significant increase in symptoms immediately after chemotherapy $(t=3.66$, $P<0.0001$ ), with a significant improvement from baseline 1 year later $(t=4.75, P<0.0001)$. A similar pattern was seen on the FACT-B scores $(F=10.24, P=0.0001, F=3.43, P=0.034$ for time and interaction, respectively). Scores in the nonchemotherapy group were generally higher and stable at the three times, whereas the chemotherapy group showed a significant improvement in quality of life at T3 $(t=5.70, P<0.0001)$. Scores on the ES scale (all three participant groups) showed a significant main effect of group $(F=3.27, P=0.041)$, time $(F=18.48, P<0.0001)$ and an interaction $(F=9.89, P<0.0001)$. Post hoc tests found no significant group differences at any time point. In both patient groups, endocrine symptoms increased significantly between T1 and T2 $(t=6.97, P=0.0001, \quad t=3.83, P<0.0001$ chemotherapy and nonchemotherapy groups, respectively) and remained high at T3.

Quality of life measures did not correlate with individual test scores at baseline, T2 or T3. Quality of life scores were not significantly lower in those participants with reliable decline on multiple measures at $\mathrm{T} 2$ or $\mathrm{T} 3$.

HRT use and treatment-induced menopause The potential effect of HRT use on cognitive function was examined across groups (the groups were balanced on the proportion of postmenopausal women who had taken HRT). There was no significant main effect of HRT use (never $v s$ current or past) on any cognitive measure at baseline and HRT use was not associated with reliable decline on multiple measures at $\mathrm{T} 2$ or T3. Patients who experienced a treatment-induced menopause (TIM) following chemotherapy treatment $(N=32$ out of 39$)$ were compared with those in the chemotherapy group who were postmenopausal at baseline. Those who had TIM were more likely to show reliable decline on multiple measures at $\mathrm{T} 2(\mathrm{OR}=2.6,95 \%$ confidence interval (CI) $0.823-8.266, P=0.086$ ) and 1.51 times as likely at T3 (95\% CI $0.405-3.922, P=0.145)$. These were not more likely to have above threshold $\mathrm{GHQ}_{12}$ scores at either time point or to report lower quality of life.

\section{DISCUSSION}

This study benefits from both a longitudinal design and the ability to control for individual difference factors such as intelligence, age and education to permit a realistic appraisal of the extent of cognitive impairment after treatment for early stage breast cancer.
Little convincing evidence was found to suggest that there is measurable and meaningful impairment for the vast majority of women in the UK who receive standard adjuvant treatments for breast cancer.

The most reliable predictor of performance on the tasks was age, intelligence and years in education. Chemotherapy, endocrine therapy, HRT use, quality of life and level of psychological distress were not associated with performance at a group or individual level. Although reliable decline on multiple measures was more common in the patient groups than in the healthy control group, there was not a significant association at either time point. More importantly, the majority of patients either showed no change or an improvement in performance. Many studies that classify patients as impaired or not, established on 'failing' a number of tests at a given criteria, are cross sectional and do not account for a change in cognitive function and other factors such as practice effects. Some of the issues relating to classifying cognitive impairment, using different statistical methods are detailed elsewhere (Shilling et al, 2005b).

A timely meta-analysis warns of the previous overinterpretation of apparent cognitive impairment in patients receiving chemotherapy. Although statistically significant cognitive impairment was found in the analysis, the magnitude of the impairment was small to moderate, with patients treated with chemotherapy performing better than the comparison group on some tests (Falleti et al, 2005). One possible reason why relatively little impairment was found in our chemotherapy group may be that the majority received relatively low dose FEC. This would support findings by van Dam et al (1998) who found no differences between patients receiving FEC (and also tamoxifen) and those not treated with systemic adjuvant therapy.

The level of psychological morbidity in the patient groups in our study was high at baseline but similar to that reported in the literature (Burgess et al, 2005). A difference between groups became apparent at the second assessment, with the chemotherapy patients maintaining high levels of psychological distress. The disparity between groups probably reflected the stage of treatment that the patients had reached. The nonchemotherapy patients had completed their radiotherapy treatment by several months, whereas many of the chemotherapy patients were waiting to begin. In common with other studies, psychological distress, quality of life measures and self-reported cognitive failures did not impact on objective tests of cognitive function, but were significantly associated with each other.

The hypothesis that women may experience greater cognitive decline if treatment results in a sudden premature menopause was supported, albeit weakly, probably due to the small sample size; these women were 2.6 times more likely to show reliable decline on multiple measures, following chemotherapy. A sudden menopause brings all the accompanying endocrine symptoms, for example hot flushes, night sweats and difficulty in sleeping. An accumulation of these may have interfered with a woman's ability to concentrate on the memory and attention tasks. The suggestion that an increase in individual endocrine symptoms may contribute significantly to cognitive performance was supported by the preliminary analysis of 50 of the present chemotherapy patients and 43 healthy controls (Shilling et al, 2005a). The most significant factor to account for the difference between the current data and that previously reported was that $52 \%$ of the patients in the preliminary analyses were premenopausal at baseline, compared to $36 \%$ in the present data set and these women were significantly more likely to experience a treatment-induced menopause (OR 3.12, $P=0.016$ ).

In summary, the results from this study suggest that only a small proportion of women receiving adjuvant treatments for breast cancer experience objective measurable change in their concentration and memory. It is reassuring that the majority are either unaffected or even improve over time, but such results are rarely emphasised in publication. The group of women who appear to be 
most at risk of exhibiting a decline in cognitive performance are those who experience a treatment-induced menopause, particularly in the initial period following chemotherapy. Future studies may like to focus on this group of women receiving treatments that induce early menopause, including LHRH therapy. This would also help clarify whether it is a reduction in oestrogen producing severe endocrine symptoms that interferes with attending to stimuli and therefore constrains memory processing, or chemotherapy treatment per se.

\section{REFERENCES}

Bender C, Sereika SM, Berga SL, Vogel VG, Brufsky AM, Paraska KK, Ryan CM (2005) Cognitive impairment associated with adjuvant therapy in breast cancer. Psycho-oncology, Aug 12 [Epub ahead of print]

Brady MJ, Cella DF, Mo F, Bonomi AE, Tulsky DS, Lloyd SR, Deasy S, Cobleigh M, Shiomoto G (1997) Reliability and validity of the Functional Assessment of Cancer Therapy-Breast quality-of-life instrument. J Clin Oncol 15: 974-986

Broadbent D, Cooper PF, FitzGerald P, Parkes KR (1982) The Cognitive Failures Questionnaire (CFQ) and its correlates. Br J Clin Psychol 21 (Part 1): $1-16$

Burgess C, Ramirez A, Cornelius V, Love S, Graham J, Richards M (2005) Depression and anxiety in women with early breast cancer: five year observational cohort study. BMJ 330: $702-705$

Falleti MG, Sanfilippo A, Maruff P, Weih L, Phillips KA (2005) The nature and severity of cognitive impairment associated with adjuvant chemotherapy in women with breast cancer: a meta-analysis of the current literature. Brain Cogn 59: $60-70$

Fallowfield LJ, Leaity SK, Howell A, Benson S, Cella D (1999) Assessment of quality of life in women undergoing hormonal therapy for breast cancer: validation of an endocrine symptom subscale for the FACT- B. Breast Cancer Res Treat 55: 189-199

Goldberg D, Williams P (1988) A User's Guide to the General Health Questionnaire. Windsor: NFER-Nelson

Golden CJ (1978) Stroop Colour and Word Test. Chicago: Stoelting Co

Jacobson NS, Truax P (1991) Clinical significance: a statistical approach to defining meaningful change in psychotherapy research. J Consult Clin Psychol 59: $12-19$

Jenkins V, Shilling V, Fallowfield LJ, Howell A, Hutton S (2004) Does hormone therapy for the treatment of breast cancer have a detrimental effect on memory and cognition? Psycho-Oncology 13: 61-66

Kneebone AC, Andrew MJ, Baker RA, Knight JL (1998) Neuropsychologic changes after coronary artery bypass grafting: use of reliable change indices. Ann Thorac Surg 65: 1320-1325

McAllister TW, Ahles TA, Saykin AJ, Ferguson RJ, McDonald BC, Lewis LD, Flashman LA, Rhodes CH (2004) Cognitive effects of cytotoxic cancer chemotherapy: predisposing risk factors and potential treatments. Curr Psychiatry Rep 6: $364-371$

Nelson H (1991) National Adult Reading Test (NART) Manual. NFERNELSON

Paganini-Hill A, Clark LJ (2000) Preliminary assessment of cognitive function in breast cancer patients treated with tamoxifen. Breast Cancer Res Treat 64: 165-176

\section{Appendix 1}

The RCI was calculated as follows:

The test-retest reliability coefficient $\left(r_{\mathrm{xx}}\right)$ was computed for each measure. The standard error of measurement $\left(\mathrm{SE}_{\mathrm{m}}\right)$ was calculated by:

$$
\mathrm{SE}_{\mathrm{m}}=\mathrm{SD}_{1}\left(\sqrt{\left[1-r_{\mathrm{xx}}\right]}\right)
$$

where $\mathrm{SD}_{1}$ is the s.d. of the baseline score. The standard error of the difference $\left(\mathrm{SE}_{\mathrm{diff}}\right)$ was calculated by:

$$
\mathrm{SE}_{\mathrm{diff}}=\sqrt{\left[2\left(\mathrm{SE}_{\mathrm{m}}\right)^{2}\right]} .
$$

The standard error of the difference describes the spread of

\section{ACKNOWLEDGEMENTS}

We thank the women who participated in the study, Jan Tuson for contributing to data collection, and all the hospital clinic and administrative staff. We also thank all of the research nurses, Rose Errington, Jayne Hughes, Sonya Mash, Helen Mitchell, Elaine Noon, Victoria Rawlins, Frances Scott, for helping recruit patients to the study. Cancer Research UK funded the study, Dr Jenkins, Dr Shilling and Mrs Morris.

Rey A (1941) Psychological examination of traumatic encephalopathy. Arch Psychol 28: 286-340

Rey A (1964) L'Examen clinique en psychologie.. Paris: Presses Universitaires de France

Sawrie SM, Chelune GJ, Naugle RI, Luders HO (1996) Empirical methods for assessing meaningful neuropsychological change following epilepsy surgery. J Int Neuropsychol Soc 2: 556-564

Schagen SB, Muller MJ, Boogerd W, Van Dam FS (2002) Cognitive dysfunction and chemotherapy: neuropsychological findings in perspective. Clin Breast Cancer 3(Suppl 3): S100-S108

Shilling V, Jenkins V, Morris R, Deutsch G, Bloomfield D (2005a) The effects of adjuvant chemotherapy on cognition in women with breast cancer-preliminary results of an observational longitudinal study. Breast 14: $142-150$

Shilling V, Jenkins V, Trapala IS (2005b) The (mis)classification of chemofog - methodological inconsistencies in the investigation of cognitive impairment after chemotherapy. Breast Cancer Res Treat 1-5, Oct 27 [Epub ahead of print]

Taylor L (1979) Psychological assessment of neurosurgical patients. In Functional Neurosurgery Marino TRAR (ed) New York: Raven Pres

Tchen N, Juffs HG, Downie FP, Yi QL, Hu H, Chemerynsky I, Clemons M, Crump M, Goss PE, Warr D, Tweedale ME, Tannock IF (2003) Cognitive function, fatigue, and menopausal symptoms in women receiving adjuvant chemotherapy for breast cancer. J Clin Oncol 21: $4175-4183$

van Dam FS, Schagen SB, Muller MJ, Boogerd W, vd Wall E, Droogleever Fortuyn ME, Rodenhuis S (1998) Impairment of cognitive function in women receiving adjuvant treatment for high-risk breast cancer: high-dose versus standard-dose chemotherapy. J Natl Cancer Inst 90: $210-218$

Wefel JS, Lenzi R, Theriault RL, Davis RN, Meyers CA (2004) The cognitive sequelae of standard-dose adjuvant chemotherapy in women with breast carcinoma: results of a prospective, randomized, longitudinal trial. Cancer 100: 2292 - 2299

Weschler D (1998) The Weschler Memory Scale-Revised Vol. 3, (edn).

Wieneke M, Dienst ER (1995) Neuropsychological assessment of cognitive functioning following chemotherapy for breast cancer. Psycho-oncology 4: $61-66$

Yellen SB, Cella DF, Webster K, Blendowski C, Kaplan E (1997) Measuring fatigue and other anemia-related symptoms with the Functional Assessment of Cancer Therapy (FACT) measurement system. J Pain Symptom Manage 13: 63-74

distribution of change scores that would be expected if no change occurred.

To establish a 90\% RC confidence interval, the $\mathrm{SE}_{\text {diff }}$ was multiplied by \pm 1.64 s.d. (Kneebone et al, 1998). These cutoff points were corrected for practice effects (Sawrie et al, 1996). The practice effect for each variable is the mean difference between the follow-up and baseline scores. Thus, for each variable, an RC $90 \%$ confidence interval was calculated by:

$$
\mathrm{RC} \text { interval }=\left(\mathrm{SE}_{\text {diff }}\right) \times( \pm 1.64)+\text { practice effect. }
$$

For each participant, a difference score was calculated representing the performance difference on each measure (T2 - T1). If this score fell outside the RC interval, a statistically significant change in performance on this measure was considered to have occurred. 